\title{
Formulation of Mupirocin Adsorbed Silver Nanoparticle with Antibiofilm Agents for Enhancing Antibacterial Activity
}

\author{
Selliamman Ravi Mahi Priya ${ }^{1}$, Rajandurai Baby Roselin ${ }^{1}$, Arjunan Karuppiah, Veintramuthu Sankar ${ }^{1, *}$ \\ Department of Pharmaceutics, PSG College of Pharmacy, Coimbatore, Tamil Nadu, INDIA.
}

\begin{abstract}
Introduction: Biofilm is a complex structure of microbiome having different bacterial colonies or single type of cells in a group, adhere to the surface to produce infections. Nanoparticles (NPs) are widely used in different technological fields especially medicine because of their antibacterial properties. Silver nanoparticles (AgNPs) possess antimicrobial and antibiofilm property against various microbial infections. Objectives: The main objective of this study was to develop new formulations and characterization of mupirocin loaded silver nanoparticles with various anti biofilm agents. Methods: Silver nanoparticles formulations were prepared by tannic acid (TA) with trisodium citrate (TSC), Para-amino salicylic acid (PASA) and ascorbic acid (ASC) as both reducing and antibiofilm agents against Staphylococcus aureus followed by surface adsorption of Mupirocin (MUP). Transmission Electron Microscope (TEM) shows that the nanoparticles are roughly spherical in shape which was also confirmed by Atomic Force Microscopy (AFM). The particle size was in the range between $50 \mathrm{~nm}-250 \mathrm{~nm}$ and found to be statistically significant $(p<0.001)$ between the three AgNP formulations. In vitro drug release using the dialysis membrane showed a maximum release of $90 \% \pm 2.343$ in AgNPs (TSC TA) formulation at the end of $7 \mathrm{hr}$ with the maximum drug entrapment of $93.75 \%$. The formulations were tested for antimicrobial activity against $S$. aureus. The results were statistically compared using one way ANOVA and student $t$ test. Conclusion: There is no significant difference in antibacterial activity between three AgNP formulations. However the best synergistic activity against planktonic $S$. aureus bacteria was shown by AgNP-PASA inspite of its minimal drug release when compared to AgNPs (TSC TA).
\end{abstract}

Key words: Staphylococcus aureus, Silver nanoparticles, Mupirocin, Surface adsorption, Antibiofilm.

\section{INTRODUCTION}

Globally, the bacterial resistance has highlighted an urgent need to battle the challenge of bacterial infection at wound sites. Antimicrobial resistance at the infected site is identified as a serious medical issue that increases morbidity as well as mortality. ${ }^{1}$ The micro-organisms can live in both the planktonic (free-living) and biofilm phenotypic states. They may play an important role in impairing healing and causing infection in both acute and chronic wounds. ${ }^{2}$ Antibiotics are preferred for the treatment of infections caused by these bacteria, because of the results achieved and their cost-effectiveness. Due to their inability of existing antibiotics to combat bacterial infections, there is a huge demand for unconventional biocides is intense.

Nanotechnology is an emerging field with enormous scope, and nanomedicine provides an excellent platform for overcoming the problem of drug resistance. ${ }^{3}$ the conjugation of antimicrobial agents and nanoparticles (NPs) also improve the capabilities to kill microbial pathogens which are the primary cause for the development of antimicrobial resistance. Nanoformulations that contain antimicrobial agents also allow
Submission Date: 12-04-2021; Revision Date: 29-05-2021; Accepted Date: 09-11-2021.

DOI: 10.5530/ijper.56.1.7 Correspondence:

Dr. $v$ Sankar Vice Principal and Professor, PSG College of Pharmacy, Peelamedu,

Coimbatore-641 004,

Tamil Nadu, INDIA.

E-mail: sansunv@yahoo. co.in

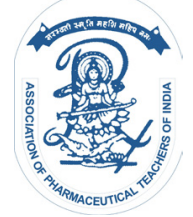

www.ijper.org 
dosage reductions and improve their activities. Antibioticconjugated NPs enhance antibiotic concentrations in sites of the bacterium - antimicrobial interaction and aid the binding of antimicrobial agents to bacteria. ${ }^{4}$ Biofilm is a complex structure of microbiome having different bacterial colonies or a single type of cells in a group that adhere to the surface of the wound. The wound infections caused by biofilms are difficult to diagnose and treat. Biofilms have detrimental effects on wound healing by affecting healing times, risk of infection and costs to health service. ${ }^{5}$

Mupirocin is an effective antibacterial agent often used in clinical practice as a topical ointment to treat a wide variety of topical wounds including burns and foot ulcers. The chemical structure consists of fatty acids that is loaded on monic acid by an ester-type linkage which mimics the carbon skeleton of isoleucine, competing with this amino acid for the active site of isoleucyltRNA bacterial synthetase, inhibiting bacterial protein synthesis. ${ }^{6}$

Silver nanoparticles (AgNPs) are one of the most leading nanoproducts for medical purposes, due to their immense antimicrobial activity. The main feature of nanoparticles is their small size, which provides a larger contact surface with the bacterial cell, increasing its penetration and enhancing their bactericidal effect. ${ }^{7}$ this involves two distinct steps that will each have an impact on its efficiency. The first one is that the ability of the system to behave in the environment of interest, where physical or chemical changes can occur. Among these, aggregation, dissolution, Redox (photo) reactions, the release of adsorbed silver species, adsorption or desorption of ions, molecular species or polymers, or interaction with other nanoparticles or surfaces can modify the speciation of silver, thereby affecting this metal availability and influencing the antibacterial effect. The next step is the way through which the silvercontaining species interact with the bacterial cell and eventually leads to cellular death. ${ }^{8}$

Tannic acid is a natural and also a process derived phenolic compound that is a potent antagonist against bacteria. The mechanism of biofilm inhibition was found to be dependent on the putative transglycosylase IsaA. ${ }^{9}$ Salicylic acid which is the main aspirin metabolite exhibits various effects on the expression of $S$. aureus virulence factors. They also possess the ability to form complexes with iron cations and it has been proved that different iron-chelating molecules reduce the formation of biofilm. They also modify the activity of CodY, a metabolite - responsive global regulator, controls metabolism and virulence and gene expression through several molecular mechanisms. It represses ica and also cap gene transcripts in $S$. aureus. ${ }^{10}$ Ascorbic acid which is commonly known as Vitamin $\mathrm{C}$ can inhibit pathogenic bacteria and inhibit biofilms. They inhibit icaA dependent polysaccharide intracellular adhesion thereby inhibiting the interaction between cells. ${ }^{11}$ The investigation was undertaken to determine the antibacterial effect of antibiofilm agents with mupirocin loaded silver nanoparticles.

\section{MATERIALS AND METHODS}

\section{Materials}

Mupirocin was obtained as a gift sample from the Pharmaceutical Company, Fourrts India Laboratories Pvt ltd, Chennai. Silver nitrate and Sodium borohydride was purchased from Sigma Aldrich.Tannic acid, Paraamino salicylic acid, Ascorbic acid, Muller-Hinton Broth, Sodium hydroxide was obtained from Himedia Laboratories Pvt. Ltd., Bangalore.

\section{Methods}

\section{Preformulation studies}

The Digital melting point apparatus was used to determine the melting point of the drug by capillary tube method. The Lambda max of mupirocin in phosphate buffer 7.4 was scanned between $200-400 \mathrm{~nm}$ by UV-Visible spectrophotometer. The calibration curve was plotted for the solution in the order of increasing concentration.

\section{Silver nanoparticle synthesis Silver nitrate reduced with sodium borohydride}

Excess sodium borohydride was needed to reduce the ionic silver and to stabilize the silver nanoparticles. Different volumes of $0.001 \mathrm{M}$ silver nitrate were added drop wise (about 1 drop per second) to $30 \mathrm{ml}$ of $0.002 \mathrm{M}$ sodium borohydride $\left(\mathrm{NaBH}_{4}\right)$ solution. This mixture was stirred on a magnetic stirrer. The solution will turn light yellow after the addition of $2 \mathrm{ml}$ of silver nitrate and then a bright yellow color develops when all of the silver nitrate has been added. The entire addition process took about 3 min and then the stirring was stopped. Reaction conditions like stirring time and quantities of reagents (both the absolute number of moles of reactant and their relative molarities) must be carefully controlled to obtain the stable yellow colloidal silver nanoparticles.

\section{Silver nitrate reduced with tannic acid}

For the preparation of silver nanoparticles, $\mathrm{AgNO}_{3}$ solution and tri-sodium citrate were used as a metal salt precursor and a reducing agent. Tannic acid (TA) solution which is a primary antibiofilm agent act as an 
auxillary reductant and stabilizer. $10 \mathrm{ml}$ of $6.8 \mathrm{mM}$ aqueous solution of tri- sodium citrate was mixed with the tannic acid solution of different concentration and heated to $60^{\circ} \mathrm{C}$ using magnetic stirrer. This mixture was added to $50 \mathrm{ml}$ solution containing $8 \mathrm{mg}$ of silver nitrate $\left(\mathrm{AgNO}_{3}\right)$ solution preheated to $60^{\circ} \mathrm{C}$ under vigorous stirring. The mixture was kept at $60^{\circ} \mathrm{C}$ for $30 \mathrm{~min}$ and boiled for 15 min respectively. The transparent colourless $\mathrm{AgNO}_{3}$ solution mixture was converted to the characteristic pale yellow colour after the addition of a required mixture of tri-sodium citrate and tannic acid solution. This color change denotes the formation of silver nanoparticle.

\section{Silver nitrate reduced with Para- Amino salicylic acid (PASA)}

Silver nitrate solution $\left(0.3 \times 10^{-3} \mathrm{~mol} / \mathrm{L}\right)$ was added drop wise to $\mathrm{pH}$ - adjusted $\mathrm{p}$-amino salicylic acid $\left(10 \times 10^{-3} \mathrm{~mol} / \mathrm{L}\right)$ in a test tube with volume ratio of $1: 1$. The mixture was then slowly heated in a boiling water bath for few minutes until it turns yellow which implies the formation of AgNPs. The mixture was cooled under tap water and transferred to a small bottle for further analysis. For reaction optimization, some parameters were varied, such as $\mathrm{pH}(10-12)$, reaction time $(5,10,15$, 20, 25, $30 \mathrm{~min})$.

\section{Silver nitrate reduced with Ascorbic acid}

Silver nitrate was used as a starting material while trisodium citrate and ascorbic acid were used as antibiofilm agent and reducing agent respectively. The concentration of trisodium citrate and ascorbic acid were varied to observe the effect of these parameters especially on the size and morphology of the silver nanoparticles. In detail, $80 \mathrm{ml}$ of $\mathrm{AgNO}_{3}$ was first heated to $60^{\circ}$ and was then added (with vigorous stirring) to $20 \mathrm{ml}$ of a trisodium citrate and ascorbic acid solution which was pre-heated to $60^{\circ}$. The mixture was then stirred for $20 \mathrm{~min}$. After that, the heating was stopped and the solution was cooled to room temperature with continuous stirring.

The solution will turn to brighter yellow/ brownish red when all of the silver nitrates had been added. During the formulation $1 \%$ of polyvinyl pyrrolidine was added as the capping agent at the end of the reaction. The spectrum peak widely ranging from 300 to $450 \mathrm{~nm}$ wave length indicates the formation of silver ions in the formulation.

\section{Surface Adsorption of Mupirocin on AgNP}

Mupirocin is adsorbed to AgNP surface by electrostatic interaction method. Mupirocin $40 \mathrm{mg}$ was added to $40 \mathrm{ml}$ of AgNP solution and stirred in a magnetic stirrer at room temperature for $24 \mathrm{hr}$. Lambda max and spectrum of the formulation after drug adsorption was determined by UV- double beam spectroscopy.

\section{Evaluation of Mupirocin loaded Silver nanoparticles}

The obtained mupirocin loaded silver nanoparticles were evaluated by their adsorption efficiency, in vitro drug release. Morphological characteristics were analyzed by Zeta sizer, Atomic Force Microscopy (AFM) and Transmission Electron Microscopy (TEM) studies. The antibacterial activity of the formulation was determined by measuring the zone of inhibition.

\section{(i) Adsorption Efficiency}

About $2 \mathrm{ml}$ of the formulation was taken in an eppendorf tube and centrifuged at $13000 \mathrm{rpm}$ for 15 min at $4^{\circ}$ and $1 \mathrm{ml}$ of the supernatant was collected, diluted with water and the absorbance was measured in UV spectrophotometer at $220 \mathrm{~nm}$ wavelength. The amount of drug adsorbed and adsorption efficiency was calculated according to the formula given below.

Amount of drug adsorbed $=$ Initial drug loaded unadsorbed drug

Adsorption efficiency $(\%)=[$ Amount of drug adsorbed/ Initial drug loaded] $\times 100$

\section{(ii) In-vitro drug release studies}

Accurately $2 \mathrm{ml}$ of the AgNP liquid formulation was taken in a dialysis bag and closed on both the sides with Hi-media closure clips to prevent the leakage of formulations. Then the dialysis bag was placed inside a beaker containing $50 \mathrm{ml}$ phosphate buffer $\mathrm{pH} 7.4$ and $\mathrm{pH} 5.8$ at room temperature and allowed to stir gently at $100 \mathrm{rpm}$ using magnetic stirrer. Samples were withdrawn at a periodic time intervals from [1-7 hr] and replaced with equal volume of buffer, then the sample was analysed in UV at $220 \mathrm{~nm}$ after making suitable dilutions and the absorbance was determined.

\section{(iii) Particle size and Zeta potential analysis for synthesized AgNP}

The mean diameter and zeta potential of the samples were measured by Dynamic light scattering (DLS) using zetasizer (Nano ZS 90, Malvern Instruments, United Kingdom). The synthesized AgNP formulations was measured in disposable polystyrene cuvettes at $25^{\circ} \mathrm{C}$ with a detection angle of $90^{\circ}$. The particle size, Polydispersivity index (PDI) and zeta potential were determined. 


\section{(iv) Morphological analysis}

Transmission Electron Microscopy (TEM 200 Kv; T12 Fei, Teenai G2 Spirit TWIN) was performed to analyze the surface morphology of AgNP formulations. The sample was placed in a copper grid and kept for air drying at room temperature. The dried sample in the grid was placed till the loading point and scanned under $200 \mathrm{Kv}$, then the beam was allowed to pass through the sample and the images were scanned under microscope at different nanometer scales.

The prepared slides were coated on the surface of the cover slip and air-dried at $28^{\circ} \mathrm{C}$ in room for $12 \mathrm{hr}$ and subjected to AFM analysis. The shape of silver nanoparticles was analyzed using an atomic force microscopy (AFM).

(v) Zone of Inhibition test for determining antibacterial activity of mupirocin loaded AgNP against Staphylococcus aureus

\section{Preparation of Mueller- Hinton (MH) agar plates}

Mueller- Hinton $(\mathrm{MH})$ agar medium was prepared according to the manufacturer's instructions and autoclaved for $20 \mathrm{~min}$ at 120 PSI. After autoclaving, the agar medium was cooled to $40-45^{\circ}$ in a water bath. The cooled agar medium was poured into a petridish and allows it to cool further at room temperature.

\section{Agar well diffusion assay}

To inoculate the $\mathrm{MH}$ agar plates, a sterile cotton swab was dipped into the bacterial suspension and swabbed over the surface of the agar plates. This procedure was repeated over three times, each time the plate was rotated approximately $60^{\circ}$ to ensure an even distribution of inoculums. Then a hole was punched aseptically with the help of sterile cork borer with a diameter of 6 to $8 \mathrm{~mm}$ with the sterile cork borer and a volume of 20-100 $\mu$ l of the antimicrobial agent/ AgNP formulations at desired concentration was introduced into the well. Amoxicillin $(10 \mu \mathrm{g})$ was used as positive control. Then the bacterial petridishes were incubated at $37^{\circ}$ for $24 \mathrm{hr}$. The sensitivity of the test organism to each antimicrobial was indicated by clear zone of inhibition around the disc and the diameter of the zone of inhibition was measured.

\section{RESULTS AND DISCUSSION}

\section{Preformulation studies}

The Melting point was determined as a part of preformulation study since it can affect the absorption of the dosage form ${ }^{12}$ and was found to be $79^{\circ} \mathrm{C}$ which complies with Indian Pharmacopoeia. From the UV-Visible spectrum lambda max of the mupirocin was optimized as $220 \mathrm{~nm}$. From the standard graph, absorbance was measured and linearity were obtained between $2-20 \mu \mathrm{g} / \mathrm{ml}$ concentration in mupirocin and the regression value was found to be $r^{2}=0.9896$. (Figure 1)

\section{Particle size and Zeta potential analysis}

Particle size of AgNP with $\mathrm{NaBH}_{4}$ as reducing agent has the particle size distribution between $113 \mathrm{~nm}$ to $243 \mathrm{~nm}$ with PDI between 0.3 to 0.5 indicating the homogeneity of the formulation. Zeta potential was found to be between $-19 \mathrm{mV}$ to $-25 \mathrm{mV}$ revealing the presence of a compact surface layer of $\mathrm{NaBH}_{4}$ on the colloidal silver. The zeta potential of the system should be between -30 to $+30 \mathrm{mV}$ to resist aggregation of nanoparticles.

Particle size of AgNP with TSC TA as reducing agent has the particle size distribution between $87 \mathrm{~nm}$ to $196 \mathrm{~nm}$ which is in accordance with the work done by katarzyna et al., using same procedure of reducing silver nitrate with tannic acid. ${ }^{13}$ with PDI between 0.2 to 0.5 indicating the homogeneity of the formulation. Zeta potential was found to be between $-33 \mathrm{mV}$ to $-27 \mathrm{mV}$ revealing the presence of a compact surface layer of TSC on the colloidal silver.

Particle size of AgNP with PASA as reducing agent has the particle size distribution between $140 \mathrm{~nm}$ to $204 \mathrm{~nm}$ which was larger than that is reported by Dian susanthy et al. synthesized AgNP using PASA ${ }^{14}$ even though the $\mathrm{pH}$ was 11 and the reaction time $20 \mathrm{~min}$. PDI between 0.3 to 0.5 indicating the homogeneity of the formulation. Zeta potential was found to be between $-22 \mathrm{mV}$ to $-25 \mathrm{mV}$. The $\mathrm{pH}$ of PASA solution and reaction time has a huge impact on stability of silver nanoparticles by altering the sedimentation rate. Increasing the $\mathrm{pH}$ of PASA might increase the reducing property but increases the particle size and decreases the stabilizing performance due to sedimentation of silver ions on storage. ${ }^{15}$ Hence in this study we have chosen the optimized AgNP formulation

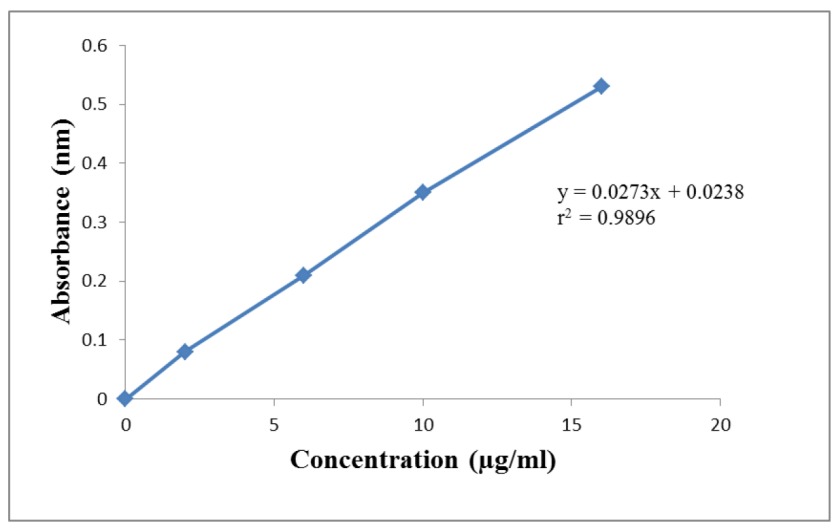

Figure 1: Standard graph of Mupirocin. 
reduced with PASA at $\mathrm{pH} 11$ with the reaction time of 20 min which resulted in good stability for 3 months.

Particle size of AgNP with ASC which is a weak reducing agent has the particle size distribution between $160 \mathrm{~nm}$ to $250 \mathrm{~nm}$ with PDI between 0.4 to 0.5 indicating the homogeneity of the formulation. Zeta potential was found to be between $-33 \mathrm{mV}$ to $-15 \mathrm{mV}$.

The solution will turn to brighter yellow/ brownish red when all of the silver nitrates had been added which is shown in Figure 2. The formulation with the lowest particle size and more stable zeta potential is mentioned in the table given below (Table 1).

Among the different formulation with different reducing agents AgNP-TSC-TA was able to produce the particles with smaller size $87.45 \mathrm{~nm}$ indicating it may improve its contact with the skin due to its smaller particle size which may provide synergistic antibacterial activity. The average particle size of $\operatorname{AgNP}\left(\mathrm{NaBH}_{4}\right)$, AgNP (TSC-TA), AgNP (PASA) and AgNP (ASC) were compared to each other and it is found to have significant $p$ value $(\phi<0.001)$. A change in the surface potential was observed after the addition of Mupirocin to AgNP. The formulation with the lowest particle size has been selected for further studies.

The resonance wavelength of AgNP formulations in UV depends on the particle size and shape. If the particle size becomes larger the plasmon peak shifts to the longer wavelength and broadens (Table 2). Thus, surface adsorption of silver nanoparticles with Mupirocin did not produce color change and caused broadening of UV spectrum peak except AgNP with ascorbic acid as it produced a marked color change and sedimentation of particles (Figure 3) indicating that ascorbic acid is

\begin{tabular}{|c|c|c|c|}
\hline \multicolumn{3}{|c|}{ Table 1: Particle size, Poly dispersity Index and Zeta } \\
Potential Characterization of AgNP. \\
\hline Formulation & $\begin{array}{c}\text { Particle } \\
\text { Size(nm) } \\
\text { Nanometer }\end{array}$ & PDI & $\begin{array}{c}\text { Zeta } \\
\text { Potential(mV) } \\
\text { millivolt }\end{array}$ \\
\hline $\begin{array}{c}\mathrm{F}_{1}(3 \mathrm{mg} \text { of } \\
\left.\mathrm{NaBH}_{4}\right)\end{array}$ & $113.3 \pm 0.665$ & $0.362 \pm 0.011$ & $-25 \pm 0.456$ \\
\hline $\begin{array}{c}\mathrm{F}_{2}(24 \mu \mathrm{M} \text { of } \\
\mathrm{TA})\end{array}$ & $87.45 \pm 0.94$ & $0.240 \pm 0.021$ & $-33.41 \pm 0.521$ \\
\hline $\begin{array}{c}\mathrm{F}_{3}(\mathrm{pH} 10) \text { of } \\
\mathrm{PASA}\end{array}$ & $148.2 \pm 0.742$ & $0.398 \pm 0.020$ & $-23.6 \pm 0.550$ \\
\hline $\begin{array}{c}\mathrm{F}_{4}(7 \mathrm{mg} \text { of } \\
\mathrm{ASC})\end{array}$ & $161.9 \pm 0.843$ & $0.428 \pm 0.023$ & $-33.1 \pm 0.429$ \\
\hline
\end{tabular}

Data was expressed as mean \pm SD $(n=3) ; p<0.001$ indicating statistical difference in the particle size between three formulations.

F - Formulation

TA - Tannic acid

PASA - Para amino salicylic acid

ASC - Ascorbic acid

$\mathrm{nm} \quad-$ Nano meter

$\mathrm{mV} \quad-$ millivolt a weak reducing agent (i.e., it cannot form silver oxide on the surface to retain its color due to the particle size of Mupirocin) thereby causing multiple peaks between 300 to $800 \mathrm{~nm}$.

\section{Surface adsorption Efficiency}

Adsorption efficiency of mupirocin loaded AgNP $\left(\mathrm{NaBH}_{4}\right), \operatorname{AgNP}$ (TSC TA) and AgNP (PASA) formulations after surface adsorption of the drug was found to be $90.5 \%, 93.5 \%$ and $89.5 \%$ (Table 3 ) and there was no color change observed in the formulation after drug adsorption. The maximum entrapment of drug on AgNP TSC TA formulation may be due to higher concentration of TA which may help to hold the drug on its surface.

\section{In vitro drug release studies}

The in vitro drug release studies was carried out in two different $\mathrm{pH}$ (i.e., 5.8 and 7.4) to check the release of the drug at highly regulated $\mathrm{pH}$ environment of skin and with the biofilm $\mathrm{pH}$. There was a burst release of Mupirocin at the first hour and the release was extended till the end of $7^{\text {th }} \mathrm{h}$ (Table 4). The in-vitro release in $\operatorname{AgNP}\left(\mathrm{NaBH}_{4}\right)$, AgNP (TSC-TA) and AgNP (PASA) formulations were statistically compared at $\mathrm{pH} 5.8$ and $\mathrm{pH} 7.4$ through one way anova (Figure 4) and the significant $p$ value $<0.005$ was observed.

\section{Morphological analysis (TEM and AFM)}

Surface morphology of AgNP formulation was carried out in TEM micrograph (Figure 5) shows that the formulations are roughly spherical in shape The size of the particles was calculated by the TEM analysis, which are mainly in range between $100-150 \mathrm{~nm}$ which confirms the report given by Dynamic Light Scattering (zeta sizer). Our TEM results are similar to the results of Rita La Spina et al. who reported the particle shape and size of AgNPs as monodispersed spherical nanoparticles

\begin{tabular}{|c|c|c|}
\hline \multicolumn{2}{|c|}{ Table 2: UV spectrum characterization of AgNP. } \\
\hline Formulation & \multicolumn{2}{|c|}{ Wavelength(nm) nanometer } \\
\hline $\begin{array}{c}\text { AgNP } \\
(\mathrm{TSC}-\mathrm{TA})+\mathrm{MUP}\end{array}$ & Before adsorption & After adsorption \\
\cline { 2 - 3 } & 420 & 450 \\
\hline $\begin{array}{c}\text { AgNP } \\
(\mathrm{NaBH} 4)+\mathrm{MUP}\end{array}$ & 410 & 415 \\
\hline $\begin{array}{c}\text { AgNP } \\
(\mathrm{PASA})+\mathrm{MUP}\end{array}$ & 415 & 430 \\
\hline
\end{tabular}

$\begin{array}{ll}\text { AgNP } & \text { - Silver nanoparticle } \\ \text { TA } & \text { - Tannic acid } \\ \text { TSC } & \text { - Trisodium citrate } \\ \text { PASA } & \text { - Para amino salicylic acid } \\ \text { nm } & \text { - Nano meter } \\ \text { MUP - Mupirocin }\end{array}$




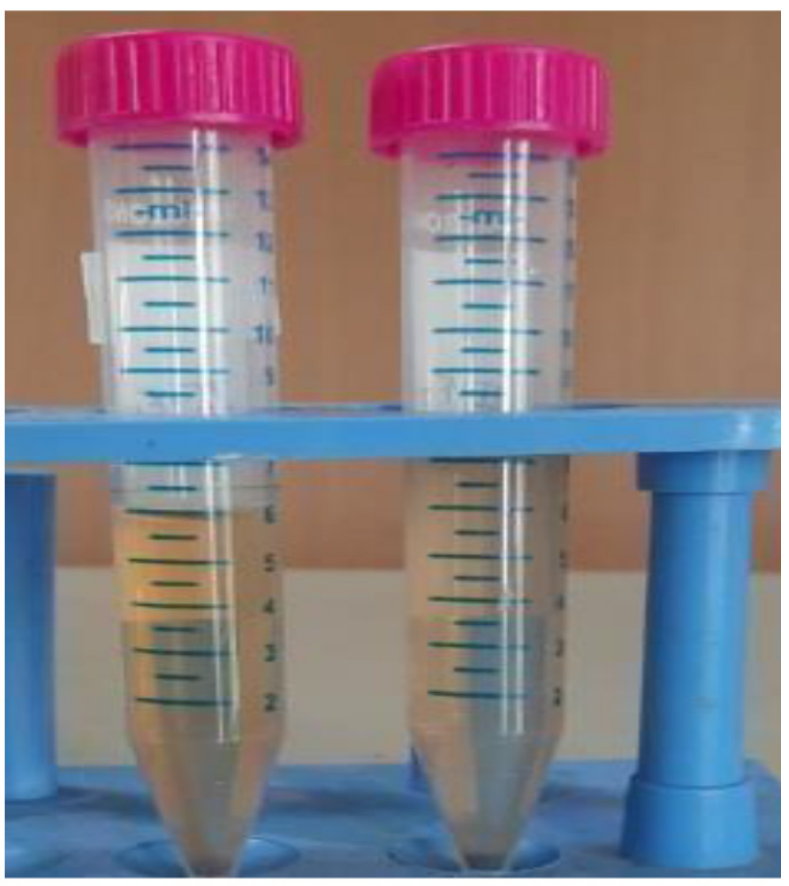

$1 \mathrm{ASC} \quad-\quad$ Ascorbic acid

\section{AgNP - $\quad$ Silver nanoparticle}

Figure 2: Images of AgNP formulation with various reducing agents.

\begin{tabular}{|c|c|}
\hline $\begin{array}{c}\text { Table 3: Entrapment efficiency of Mupirocin on } \\
\text { AgNP. }\end{array}$ \\
\hline Formulation & Entrapment efficiency \\
\hline AgNP(NaBH $\left.{ }_{4}\right)$ & $90.5 \%$ \\
\hline$A g N P(T S C+T A)$ & $93.5 \%$ \\
\hline AgNP (PASA) & $89.5 \%$ \\
\hline
\end{tabular}

AgNP - Silver nanoparticle

TA - Tannic acid

TSC - Trisodium citrate

PASA - Para amino salicylic acid

with the size range of $100-200 \mathrm{~nm} .{ }^{16}$ The spherical surface morphology was confirmed by two dimensional AFM and the height of the nanoparticles is in the range of $28-38 \mathrm{~nm}$ (Figure 5).

\section{Zone of Inhibition}

Bacterial sensitivity test was carried out for clinical $S$. aureus strain. Mupirocin is more effective against $S$. aureus than the other antibiofilm agent (Tannic acid and PASA) when tested alone (Table 5). Among the two antibiofilm agents PASA showed better antimicrobial activity. Inspite of its slow drug release on in vitro studies, AgNP-PASA seems to have more antibacterial activity against $S$. aureus.
Table 4: In vitro drug release data at the end of seventh hour.

\begin{tabular}{|c|c|c|c|}
\hline \multirow{2}{*}{ S.No } & \multirow{2}{*}{ Formulation } & \multicolumn{2}{|c|}{ In vitro drug release } \\
\cline { 3 - 4 } & & $\mathbf{p H ~ 5 . 8}$ & $\mathbf{p H ~ 7 . 4}$ \\
\hline 1. & $\mathrm{AgNP}\left(\mathrm{NaBH}_{4}\right)$ & $85 \% \pm 3.566$ & $87 \% \pm 0.5657$ \\
\hline 2. & $\mathrm{AgNP}(\mathrm{TSC}+\mathrm{TA})$ & $90 \% \pm 2.434$ & $89 \% \pm 2.343$ \\
\hline 3. & $\mathrm{AgNP}(\mathrm{PASA})$ & $86 \% \pm 1.434$ & $86 \% \pm 1.456$ \\
\hline
\end{tabular}

Data was expressed as mean $\pm \mathrm{SD}(n=3) ; p<0.005$ indicating statistical difference in the in vitro between three formulations.
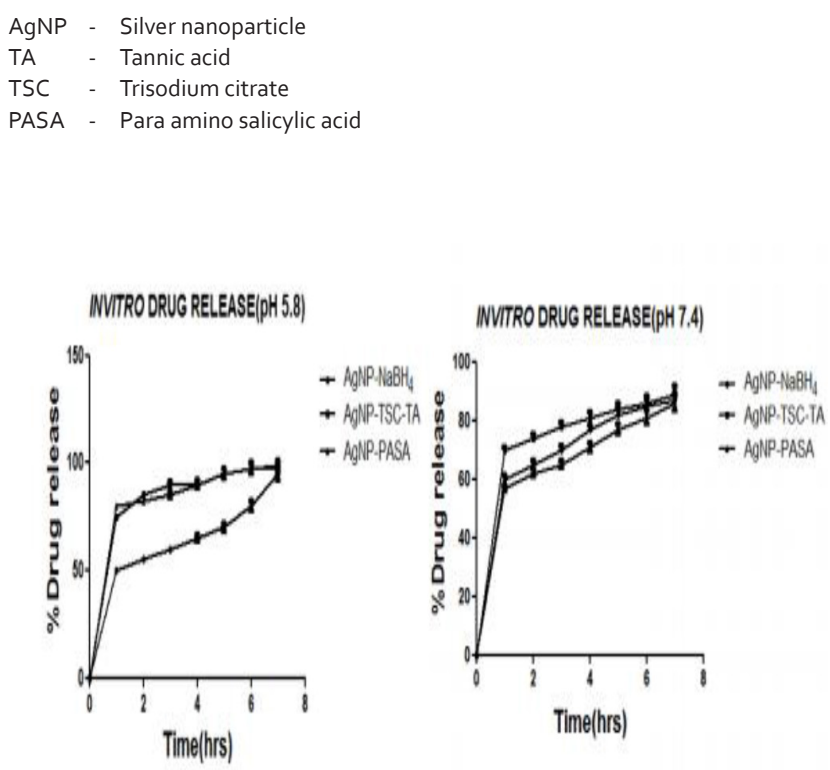

$$
\begin{array}{lll}
\text { TSC } & - & \text { Trisodium citrate } \\
\text { TA } \quad- & \text { Tannic acid } \\
\text { PASA - } & \text { Para amino salicylic acid }
\end{array}
$$

Figure 3: In vitro drug release at pH 5.8 and pH 7.4.

Statistically significant difference between the antibacterial activity of mupirocin vs tannic acid and para amino salicylic acid were analyzed by one way ANOVA test. Mupirocin has significant $(p<0.0001)$ antibacterial activity than the other two antimicrobial agents. Statistically significant difference between the antibacterial activity of tannic acid and para amino salicylic acid were analyzed by student $\mathrm{T}$ test which concluded that there is no significant $(p>0.0001)$ difference between the two antibiofilm agents.

Bacterial sensitivity test for the synthesized AgNP formulation was carried out for clinical $S$. aureus strain (Table 6). The formulation that contains PASA is more effective against $S$. aureus than the one which had tannic acid.

Hence this study revealed that Mupirocin loaded silver nanoparticles with antibiofilm agents has good antibacterial acivity than mupirocin alone. 


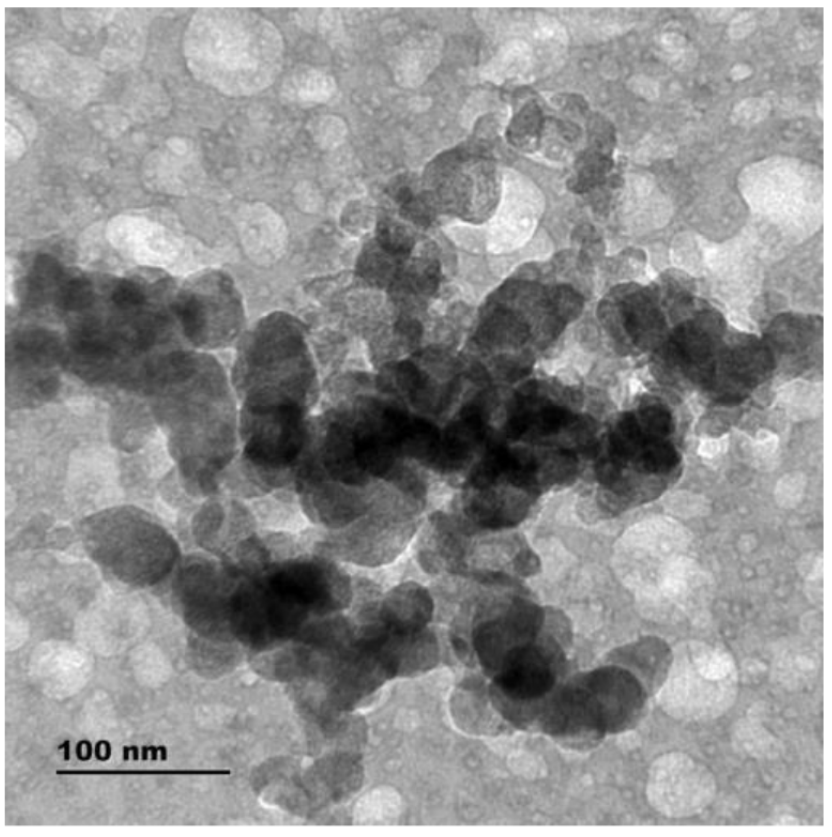

\section{AgNP - $\quad$ Silver nanoparticle \\ TEM - Transmission electron microscopy}

TA - Tannic acid

TSC - Trisodium citrate

Figure 4: TEM results of AgNP (TSC-TA).

\begin{tabular}{|c|c|c|}
\hline \multicolumn{2}{|c|}{ Table 5: Zone of inhibition against S. aureus. } \\
\hline S.No & Formulation & $\begin{array}{c}\text { Zone of Inhibition } \\
(\mathbf{m m})\end{array}$ \\
\hline 1 & Mupirocin & $30 \pm 0.563$ \\
\hline 2 & Tannic acid & $12 \pm 0.450$ \\
\hline 3 & Para amino Salicylic acid & $18 \pm 0.521$ \\
\hline 4 & $\begin{array}{c}\text { Positive } \\
\text { Control(Amoxicillin) }\end{array}$ \\
\hline
\end{tabular}

Data was expressed as mean $\pm \operatorname{SD}(n=3) ;(p<0.0001)$ indicating statistical difference in the zone of inhibition between mupirocin and antibiofilm agents.

S.aureus - Staphylococcus aureus

$\mathrm{mm}$ - millimeter

\section{CONCLUSION}

In the present work silver nanoparticles were synthesized by chemical reduction method using sodium borohydride, tri sodium citrate tannic acid, Para amino salicylic acid as reducing agents and drug mupirocin was surface adsorbed to the formulation. The particle size was in size range of $50 \mathrm{~nm}-250 \mathrm{~nm}$. The zeta potential was in the range of $-15 \mathrm{mV}$ to- $-50 \mathrm{mV}$ and PDI values ranging from $0.2-0.7$. In vitro drug release using dialysis membrane showed maximum release of $90 \% \pm 2.343$ in $\mathrm{AgNP}$ (TSC TA) formulation at $7 \mathrm{hr}$ with the maximum drug entrapment of $93.75 \%$.

\begin{tabular}{|c|c|c|}
\hline \multicolumn{3}{|c|}{ Table 6: Zone of inhibition of AgNPs against } \\
S. aureus. \\
\hline S.No & Formulation & $\begin{array}{c}\text { Zone of } \\
\text { Inhibition }(\mathbf{m m})\end{array}$ \\
\hline 1 & $\mathrm{AgNP}\left(\mathrm{NaBH}_{4}\right)$ & $35 \pm 0.429$ \\
\hline 2 & AgNP + Tannic acid & $36 \pm 0.545$ \\
\hline 3 & AgNP +Para amino Salicylic acid & $37 \pm 0.412$ \\
\hline
\end{tabular}

Data was expressed as mean \pm SD $(n=3) ;(p>0.0001)$ indicating no statistical difference in the antibacterial activity between three formulations.

AgNP - Silver nanoparticle

S.aureus - Staphylococcus aureus

$\mathrm{mm} \quad-$ millimeter

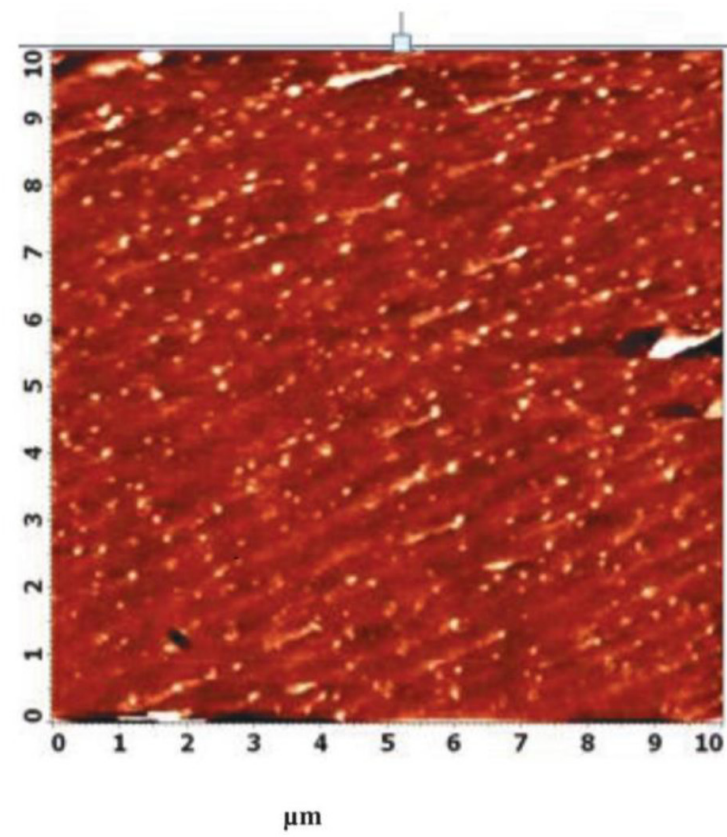

AgNP - $\quad$ Silver nanoparticle

AFM - $\quad$ Atomic Force microscopy

TA - Tannic acid

TSC - Trisodium citrate

Figure 5: AFM results of AgNP (TSC-TA).

The formulations were tested for antimicrobial activity against $S$. aureus and almost all the combination showed effective and synergistic antimicrobial activity. The best synergistic activity against planktonic $S$. aureus bacteria was shown by AgNP- PASA inspite of its minimal drug release when compared to AgNP (TSC TA). Hence this combination can be subjected for further studies to confirm the eradication against biofilm.

\section{ACKNOWLEDGEMENT}

The authors would like to thank Fourrts India Laboratories Pvt Ltd, Chennai for providing the gift samples of mupirocin respectively. We gratefully acknowledge our principal Dr. M. Ramanathan 
M.Pharm, Ph.D., for providing the research facilities throughout this work. We also extend our thanks to our institution PSG College of Pharmacy, Coimbatore for the huge facilities offered for completing of our project. We acknowledge Council of Scientific and Industrial Research, New Delhi for providing major extramural research grant to initiate the preliminary research work (Grant. No: 60(0117)/19/EMR-II). We also acknowledge The Tamil Nadu Dr.M.G.R. Medical University, Chennai, India for providing minor research grant.

\section{CONFLICT OF INTEREST}

The authors declare no conflict of interest.

\section{ABBREVIATIONS}

AgNP: Silver Nanoparticles; TA: Tannic acid; TSC: Trisodium citrate; PASA: Para aminosalicylic acid; ASC: Ascorbic acid; MH: Mueller-Hinton; AFM: Atomic force microscopy; TEM: Transmission Electron Microscopy.

\section{REFERENCES}

1. Shaikh Sibhghatulla, Fatima Jamale, Shakil Shazi, Rizvi Syed Mohd Danish, Kamal Mohammad Amjad. Antibiotic resistance and extended spectrum beta-lactamases: Types, epidemiology and treatment. Saudi J Biol Sci. 2015;22(1):90-101. doi: 10.1016/j.sjbs.2014.08.002, PMID 25561890.

2. Qureshi M, Asif N, Baig S. Evaluation of extended spectrum -lactamase mediated resistance in Escherichia coli and Klebsiella in urinary tract infection at a tertiary care hospital. Biomedica. 2013;29:78-81.

3. Sooresh Aishwarya, Kwon Hyeogsun, Taylor Robert, Pietrantonio Patricia, Pine Michelle, Sayes Christie M. Surface functionalization of Silver Nanoparticles: Novel Applications for Insect vector Control. ACS Appl Mater Interfaces. 2011;3(10):3779-87. doi: 10.1021/am201167v, PMID 21957003.
4. Pansara Chintan, Chan Wei Yee, Parikh Ankit, Trott Darren J, Mehta Tejal, Mishra Renuka, et al. Formulation Optimization of Chitosan-Stabilized Silver Nanoparticles using in vitro Antimicrobial Assay. J Pharm Sci. 2019;108(2):1007-16. doi: 10.1016/j.xphs.2018.09.011, PMID 30244012.

5. AmmarAlguburi Nicole Comito, DimitriKashtanov, Leon MT. Dicks. Control of biofilm formation: Antibiotics and beyond. Appl Environ Microbiol. 2017;83:37.

6. Parenti MA, Hatfield SM, Leyden JJ. Mupirocin: A topical antibiotic with a unique structure and mechanism of action. Clin Pharm. 1987;6(10):761-70. PMID 3146455.

7. Sangiliyandi Gurunathan Jae Woong Han, Kwon Deug Nam. Enhanced antibacterial and anti-biofilm activities of silver nanoparticles against gramnegative and gram-positive bacteria. Nanoscale Res Lett. 2014;9:373-90.

8. Remziyeguzel GulbaharErdal. Synthesis of silver nanoparticles, fabrication and characterization.intech open. 2018;3:1-18.

9. Payne David E, Nicholas R, Martin Katherine R, Parzych Alex H, Rickard Adam Underwood, et al. Tannic Acid Inhibits Staphylococcus aureus Surface Colonization in an IsaA-Dependent Manner. Infect. Immunol J. 2013;81(2):496-504.

10. LattabAicha DjibaouiRachid, Arabi Abed DahahHichem. Effect of salicylic acid on biofilm formation and on some virulence factors in Pseudomonas aeruginosa. Int J Pharm Sci. 2017;10(2):60-71.

11. Verghese Rohan Jacob, Mathew Stephen K, David Alice. Antimicrobial activity of Vitamin C demonstrated on uropathogenic Escherichia coli and Klebsiella pneumoniae. J Curr Res Sci Med. 2017;3(2):88-93. doi: 10.4103/ jcrsm.jcrsm_35_17.

12. Chu Katherine A, Yalkowsky Samuel H. An interesting relationship between drug absorption and melting point. Int J Pharm. 2009;373(1-2):24-40. doi: 10.1016/j.ijpharm.2009.01.026, PMID 19429285.

13. Ranoszek-Soliwoda Katarzyna, Tomaszewska Emilia, Socha Ewelina, Krzyczmonik Pawel, Ignaczak Anna, Orlowski Piotr, et al. The role of tannic acid and sodium citrate in the synthesis of silver nanoparticles. J Nanopart Res. 2017;19(8):273. doi: 10.1007/s11051-017-3973-9, PMID 28824288.

14. Susanthy D, Juari Santosa S, Sri Kunarti E. The performance of p-aminosalicylic acid as reducing and stabilizing agent in silver nanoparticles Synthesis. Orient J Chem. 2019;35(1):56-63. doi: 10.13005/ojc/350106.

15. Aji A, Kunarti ES, Santosa SJ. Synthesis of Gold Nanoparticles Using $<i>p<1$ i>-Aminobenzoic Acid and <i>p</i>-Aminosalicylic Acid as Reducing Agent. Indones J Chem;19(1). doi: 10.22146/ijc.26839.

16. La Spina Rita, Mehn Dora, Fumagalli Francesco, Holland Margaret, Reniero Fabiano, Rossi François, Gilliland Douglas Synthesis of Citrate-Stabilized Silver Nanoparticles Modified by Thermal and $\mathrm{pH}$ Preconditioned Tannic Acid. Nanomaterials (Basel). 2020;10(10). doi: 10.3390/nano10102031. PMID 33076398.

\section{PICTORIAL ABSTRACT}

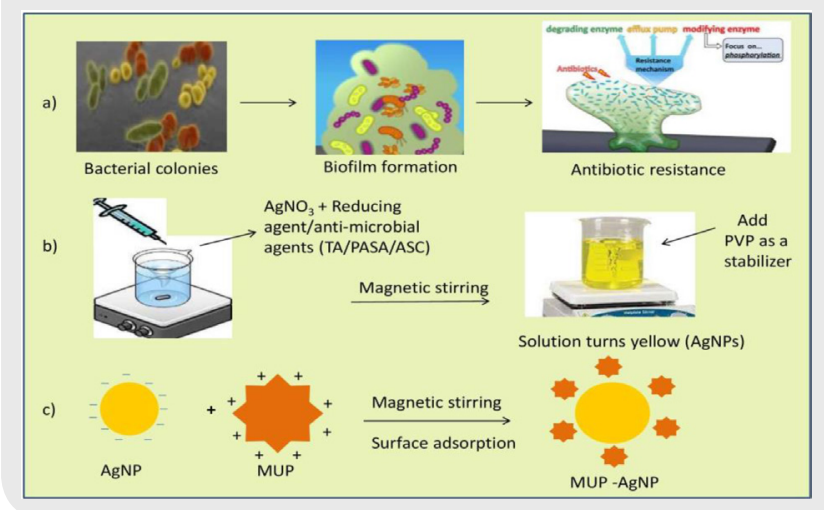

\section{SUMMARY}

- The rapid emergence in bacterial resistance is alarming worldwide, endangering the activity of antibiotics. Hence this research work would provide an alternative approach to conventional antibiotic treatment for infection control by employing novel nanoparticles to combat the resistance through biofilms.

Cite this article: Priyaa SRM, Roselin RB, Arjunan K, Sankar V. Formulation of Mupirocin Adsorbed Silver Nanoparticle with Antibiofilm Agents for Enhancing Antibacterial Activity. Indian J of Pharmaceutical Education and Research. $2022 ; 56(1): 50-7$. 